\title{
An axisymmetric PFEM formulation for bottle forming simulation
}

\author{
Pavel B. Ryzhakov
}

Received: date / Accepted: date

\begin{abstract}
A numerical model for bottle forming simulation is proposed. It is based upon the Particle Finite Element Method (PFEM) and is developed for the simulation of bottles characterized by rotational symmetry. The PFEM strategy is adapted to suit the problem of interest. Axisymmetric version of the formulation is developed and a modified contact algorithm is applied. This results in a method characterized by excellent computational efficiency and volume conservation characteristics. The model is validated. An example modelling the final blow process is solved. Bottle wall thickness is estimated and the mass conservation of the method is analyzed.
\end{abstract}

Keywords glass manufacturing · numerical modelling $\cdot$ Lagrangian · axisymmetric · final blow

\section{Introduction}

The history of bottle manufacturing counts more than 2500 years. The invention of the automatic glass bottle blowing machine in 1903 led to a true technological break-through [1], [2]. It allowed for mass production of glass bottles or jars with uniform height, weight, and capacity. This turned the bottles into the commodity it has become today. The modern automated production lines can produce over million bottles a day [3].

A standard bottle manufacturing process is sketched in Fig. 1.

First, a stream of molten glass (at around $1500{ }^{\circ} \mathrm{C}$ in case of using soda-limesilica $\mathrm{Na}_{2} \mathrm{O}-\mathrm{CaO}-\mathrm{SiO}_{2}$ glass, a common material for the bottles) is cut with a shearing blade into gobs of predetermined weight. Thes gobs are dropped into the first mould as shown in Fig. 1 a), where the temperature drops to some 1200

P. Ryzhakov

Centre Internacional de Mètodes Numèrics en Enginyeria (CIMNE)

Gran Capitán s/n, 08034 Barcelona, Spain

Tel.: +34 934017399

E-mail: pryzhakov@cimne.upc.edu 
a)
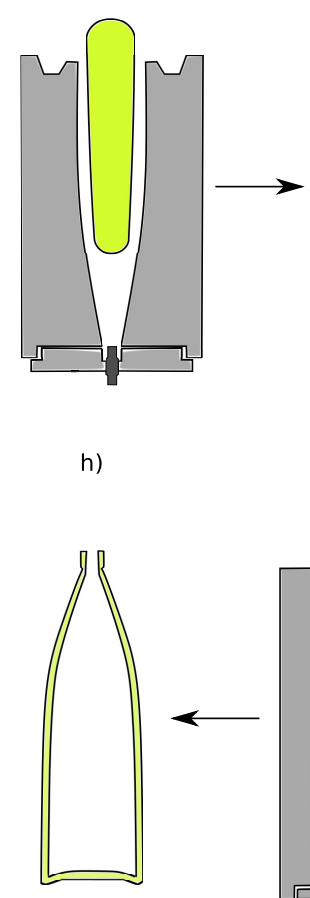

b)

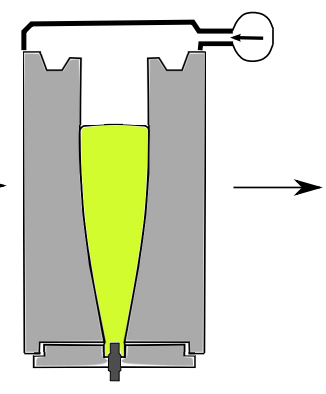

g)

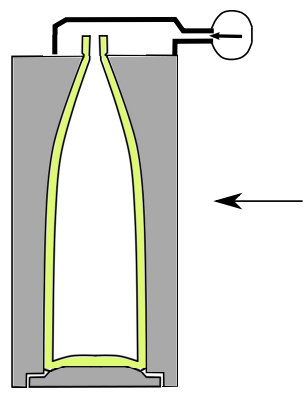

c)

d)

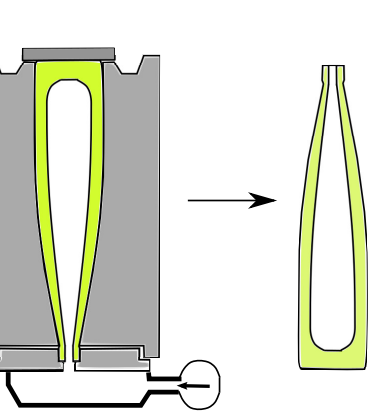

f)

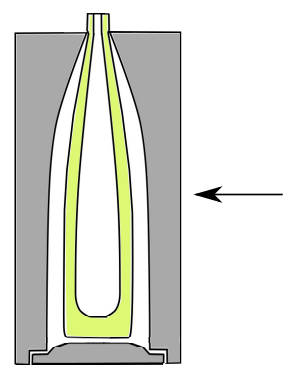

e)

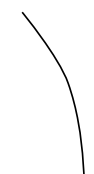

Fig. 1 Different stages of bottle manufacturing process

${ }^{\circ} \mathrm{C}$. Next, compressed air or a plunger pushes the gob to the bottom of the mould (Fig. 1 b)) and the bottle neck shape is created. Afterwards, compressed air $(\approx$ $0,15 \mathrm{MPa}$ ) is applied from the bottom of the mould forcing the gob to rise and assume the shape of the mould (Fig. $1 \mathrm{c}$ )). This stage is known as the counter-blow process, suggesting that the air acts in the direction opposite to that of gravity. The product of the counter-blow process is a thick-walled preform known as parison.

Next, the object is transferred by the robotic arm to the second mould (Fig. 1 d)). There, turned upside-down and hung by the bottleneck, it undergoes stretching due to gravity (Fig. 1 e)), until (or pior to) the contact with the mould bottom is established. Then air pressure is used to inflate the preform leading to the ultimate shape of the bottle (Fig. $1 \mathrm{f}$ )). This stage is known as the final blow process. The bottle is then removed from the mould and is transferred to the annealing furnace where it is reheated to remove the residual stresses produced during forming and is finally cooled to the ambient temperature. The forming process, from the time when the gob is dropped into the first mould until the final product is removed from the second mould takes only a few seconds.

Even though glass bottle manufacturing can be considered a well-established industrial technique up-to-date, growing competition calls for continuous improvements of the industrial process with the aim of reducing costs and improving the reliability of the containers. Moroever, development of bottles with new shapes (this is common, in particular, in perfume industry) requires to design adequate moulds and establish new operation conditions. The "optimal" operation condi- 
tions must ensure desirable wall thickness distribution and prevent concentrations of residual stresses. Surprisingly, to a great extent, the choice of the operation conditions still relies primarily upon the craftsmanship and trial-and-error rather than scientific knowledge. Industries that are producing bottles with new shapes on demand typically develop manufacture multiple parison moulds and perform the corresponding tests until satisfactory thickness distribution in the final product is obtained.

Numerical modelling is a promising alternative to the trial-and-error approach to bottle manufacturing. However, relatively few efforts devoted to numerical modelling and simulation of bottle forming have been paid ([4], [5], [6], [?], [7]). General purpose CFD software (typically based upon fixed-grid Eulerian meshes) have not proven to be easily adapted to the problem of interest. To the best of our knowledge, the only problem-specific commercial code available on the market is NOGRID PointsBlow [8], which is based upon a 3D mesh-free Lagrangian formulation. Being commercial, neither the details of the employed numerical formulation nor the possibility of extending and enriching this software exists. Therefore, a substantial need for an open-source model is evident.

From the modelling perspective, bottle forming is a complex thermo-mechanical process. It is challenging as the governing equations are strongly coupled and nonlinear. The necessity of an accurate tracking of the deforming glass domain makes it even more challenging. The difficulty is increased due to the fact that the mesh resolution must be sufficiently fine in order to discretize the bottle wall thickness in an acceptable way. Keeping such mesh resolution in the entire computational domain leads to hundreds of thousands or even millions of grid points in case of using truly 3-dimensional (3D) models. Up-to-date 3D models are characterized by very high computational costs which precludes their use by the bottle manufacturing industries. Luckily, many bottles are characterized by rotational symmetry. In such cases a feasible modelling option are the axisymmetric formulations.

In the present work a Lagrangian axisymmetric model is developed and implemented. It is based upon the Particle Finite Element Method (PFEM) [9]. PFEM is a method that has proven to be advantageous for modelling problems with moving/deforming boundaries and interfaces [10], [11]. This work presents the first axisymmetric version of the PFEM. Moreover, the model is equipped with a simple yet accurate contact algorithm. The model is implemented within an open source multi-physics code KRATOS [12], [13].

The paper presents the governing equations both at continuum and discrete level. The solution algorithm is presented. The model is validated using an example with a known analytic solution. The paper concludes with a numerical example modelling the final blow stage of the bottle manufacturing process. The aim of the paper is to show that PFEM can be an advantageous methodology for the simulation of bottle manufacturing process. In the present work thermal coupling is neglected and the mechanical model is used assuming constant viscosity. Thermal coupling will be developed and implemented in the next step of our developments. Clearly, in order to carry out predictive simulations of practical interest it is essential to introduce the thermo-mechanical coupling. This is necessary in order to compute strongly temperature-dependent viscosity of the glass [14]. 


\section{Numerical model}

Kinematic framework Lagrangian description for modelling the glass forming processes appears attractive as it allows to track the evolution of the deforming domain naturally. The position of the evolving domain coincides with the position of the mesh nodes, defined by the solution of the flow problem. Accurate interface capturing using fixed-grid (Eulerian) approaches (such as Level Set [15] or Volume of Fluid [16] ) would require much higher mesh resolution to achieve similar precision. Moreover, Level Set approach often exhibits poor mass conservation [17], which is unacceptable for the problem of interest. Lagrangian models lead to accurate boundary tracking. However, they introduce an additional computational cost due to the necessity of re-meshing the computational domain in order to avoid excessive element distortion.

The Particle Finite Element Method (PFEM) applied in the present work for modelling the glass forming is based on the updated Lagrangian description of the governing equations [10]. The key idea of the PFEM is that the variables of interest are stored at the nodes instead of the Gauss points. A finite element mesh is created at every time step of the transient problem and the solution is then stored at the nodes. The nodes are generally maintained (unless adaptive refinement or erasal is performed), thus the mesh re-generation consists in reconnecting the existing nodes. The nodes move according to their velocity obtaining their new position and then the finite element mesh is re-generated using an unconstrained Delaunay triangulation [18], [19]. The discrete operators are updated at every non-linear iteration step according to the newly obtained domain configuration, ensuring excellent convergence of the iterative procedure.

In the following the governing equations for the glass are specified. Air is neglected, thus the glass-air interface becomes simply the glass domain boundary. The fully sticking contact with the mould is considered. Thermal coupling is neglected. In the Section 2.2 the issues related to the contact treatment are explained in detail.

\subsection{Governing equations}

At forming temperatures, elastic effects in the glass are negligible and the behavior is nearly iscochoric. Thus, hot glass can be modelled as a viscous incompressible fluid governed by the incompressible Navier-Stokes equations. These can be defined on the domain $\Omega$ as:

$$
\begin{array}{r}
\rho \frac{D \mathbf{v}}{D t}+\nabla p-\nabla \cdot(2 \mu \dot{\epsilon}(\mathbf{v}))=\rho \mathbf{g} \\
\nabla \cdot \mathbf{v}=0
\end{array}
$$

where $\mathbf{v}$ is the velocity vector, $p$ the pressure, $t$ - time, $\mathbf{g}$ the body force, $\rho$ the density, $\mu$ the dynamic viscosity and $\dot{\epsilon}=\frac{\nabla \mathbf{v}+\nabla^{T} \mathbf{v}}{2}$ - the deviatoric strain rate.

At the fixed domain parts (e.g. mould walls) $\Gamma_{d}$ homogeneous Dirichlet boundary conditions are prescribed:

$$
\mathbf{v}=0 \quad \text { at } \Gamma_{d}
$$




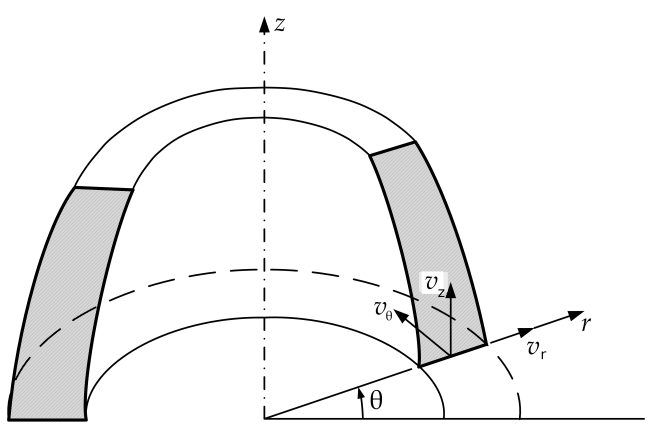

Fig. 2 An axisymmetric problem and cylindrical coordinates

Two types of Neumann boundary conditions will be distinguished. First one is the "free-surface" condition that can be approximated for vanishing velocity gradients as: $p=0$ at $\Gamma_{n}^{0}$. This condition is prescribed at the evolving outer surface of the glass prior to the contact with the mould and at the inner surface prior to the application of the compressed air. Second type of Neumann condition will be used in order to account for the inlet air pressure $p_{i}$ at $\Gamma_{n}^{p}$ :

$$
\mathbf{t}_{p}=p_{i} \mathbf{n} \quad \text { at } \Gamma_{n}^{p}
$$

This condition is prescribed at the inner surface of the glass domain. In the case of not accounting for the air pressure, the entire Neumann boundary will be considered "free-surface".

\subsubsection{Axisymmetric equations}

For obtaining an axisymmetric model one must re-write the Navier-Stokes equations in terms of cylindrical coordinates $r, z, \theta$. Axisymmetric settings are shown in Fig. 2.

Since the circumferential velocity is zero due to the revolution symmetry, only two velocity components remain: $\mathbf{v}_{a}=\left[\begin{array}{lll}\mathbf{v}_{r}(r, z) & \mathbf{v}_{z}(r, z)\end{array}\right]^{T}$ (index $a$ stands for "axisymmetric"). Pressure can be expressed as $p_{a}=p(r, z)$ and gravity as $\mathbf{g}_{a}=$ $\left[\begin{array}{ll}0 & -9.8\end{array}\right]^{T} \mathrm{~m} / \mathrm{s}^{2}$. Even though circumferential velocity is zero, circumferential strain rates are different than zero: the points situated on a circumference at radius $r$ prior to deformation move to the position $r+u$ after deforming, where $u$ is the axial displacement. This leads to a circumferential strain rate $\dot{\epsilon}_{\theta}=\frac{\mathbf{v}_{r}}{r}$. Thus the axisymmetric strain rate vector is defined as:

$$
\dot{\epsilon}_{a}=\left(\begin{array}{c}
\dot{\epsilon}_{r} \\
\dot{\epsilon}_{z} \\
\dot{\epsilon}_{\theta} \\
\dot{\gamma}_{r z}
\end{array}\right)=\left(\begin{array}{c}
\frac{\partial \mathbf{v}_{r}}{\partial r} \\
\frac{\partial \mathbf{v}_{z}}{\partial z} \\
\frac{\mathbf{v}_{r}}{r} \\
\frac{\partial \mathbf{v}_{r}}{\partial z}+\frac{\partial \mathbf{v}_{z}}{\partial r}
\end{array}\right)
$$

The axisymmetric Navier-Stokes equations can thus be written as:

$$
\begin{array}{r}
\rho \frac{D \mathbf{v}_{a}}{D t}+\nabla p_{a}-\nabla \cdot\left(2 \mu \dot{\epsilon}_{a}\left(\mathbf{v}_{a}\right)\right)=\rho \mathbf{g}_{a} \\
\nabla \cdot \mathbf{v}_{a}=0
\end{array}
$$




\subsubsection{Finite Element axisymmetric formulation}

Equal order linear velocity-pressure Finite Element shape functions over 3-noded triangles are used here for the space discretization of the primary variables in equations Eqs. (6), (7). We assume Backward Euler time discretization scheme exclusively for the sake of simplicity. All the arguments presented in the paper are valid for any implicit time integration scheme. Being standard, the space and time discretization are not discussed here (see e.g. [20] or [21] (Vol.3)).

Given $\overline{\mathbf{v}}_{n}$ and $\overline{\mathbf{p}}_{n}{ }^{1}$ at $t_{n}$, the time discrete problem consists in finding $\overline{\mathbf{v}}_{n+1}$ and $\overline{\mathbf{p}}_{n+1}$ at $t_{n+1}$ as the solution of

$$
\begin{aligned}
& \mathbf{M} \frac{\overline{\mathbf{v}}_{n+1}-\overline{\mathbf{v}}_{n}}{\Delta t}+\mathbf{C} \overline{\mathbf{v}}_{n+1}+\mathbf{G} \overline{\mathbf{p}}_{n+1}=\overline{\mathbf{F}} \\
& \mathbf{G}^{T} \overline{\mathbf{v}}_{n+1}+\mathbf{S} \overline{\mathbf{p}}_{n+1}=0
\end{aligned}
$$

where $\mathbf{M}$ is the mass matrix, $\mathbf{L}$ is the Laplacian matrix, $\mathbf{G}$ is the gradient matrix, $\overline{\mathbf{v}}$ and $\overline{\mathbf{p}}$ are the velocity and pressure respectively and $\overline{\mathbf{F}}$ is the body force vector. Note the absence of the convective term due to adopting Lagrangian kinematic framework. Pressure stabilization $\mathbf{S}$ term is added due to the use of the equal order velocity-pressure formulation [22].

The matrices and the vectors in Eqs. (8), (9) are assembled from the elemental contributions defined as

$$
\begin{aligned}
& \mathbf{M}=2 \pi \rho \iint_{A} r \mathbf{N N}^{T} d A \\
& \mathbf{C}=2 \pi \mu \iint_{A} r \mathbf{B}^{T} \mathbf{B} d A \\
& \mathbf{G}=-2 \pi \iint_{A} r \nabla \mathbf{N} \mathbf{N} d A \\
& \overline{\mathbf{F}}=2 \pi \iint_{A} r \mathbf{N} \rho \mathbf{g} d A+2 \pi \int_{\Gamma_{n}} r \mathbf{N} p_{i} \mathbf{n} d \Gamma_{n} \\
& \mathbf{S}=2 \pi \tau \iint_{A} r \nabla \mathbf{N} \nabla \mathbf{N}^{T} d A
\end{aligned}
$$

where

$$
\mathbf{B}=\left(\begin{array}{cccccc}
\frac{\partial N_{1}}{\partial r} & 0 & \frac{\partial N_{2}}{\partial r} & 0 & \frac{\partial N_{3}}{\partial r} & 0 \\
0 & \frac{\partial N_{1}}{\partial z} & 0 & \frac{\partial N_{2}}{\partial z} & 0 & \frac{\partial N_{3}}{\partial z} \\
\frac{N_{1}}{r} & 0 & \frac{N_{2}}{r} & 0 & \frac{N_{3}}{r} & 0 \\
\frac{\partial N_{1}}{\partial z} & \frac{\partial N_{1}}{\partial r} & \frac{\partial N_{2}}{\partial z} & \frac{\partial N_{2}}{\partial r} & \frac{\partial N_{3}}{\partial z} & \frac{\partial N_{3}}{\partial r}
\end{array}\right)
$$

$\mathbf{N}$ stands for the vector of standard linear FE shape functions, $A$ is the area of the element, $\tau$ is an algorithmic stabilization coefficient defined as $\tau=\left(\frac{2\|\overline{\mathbf{v}}\|}{h}+\frac{4 \nu}{h^{2}}\right)^{-1}$, where $h$ is the element size. Note that discrete operators given by Eqs. (10)-(14)

1 Note that $\overline{\mathbf{v}}=\overline{\mathbf{v}}_{a}=\left[\overline{\mathbf{v}}_{r}(r, z) \quad \overline{\mathbf{v}}(r, z)\right]^{T}$ and $\overline{\mathbf{p}}=\overline{\mathbf{p}}_{a}=\overline{\mathbf{p}}_{a}(r, z)$ are the discrete counterparts $\mathbf{v}_{a}$ and $p_{a}$ respectively. We shall omit the $a$ index in what follows. 
correspond to the unknown current configuration $\mathbf{X}_{n+1}$ according to updated Lagrangian approach [10], [23]. Thus, the system is non-linear and must be solved in an iterative manner updating discrete operators at every non-linear iteration.

Note that the volume differential can be expressed as $d V=(r d \theta) d r d z$ for axisymmetric problems. Thus, an integral of an integrand $\psi$ can be written as follows $\iiint_{V} \psi d V=\iint_{A} \int_{0}^{2 \pi} \Psi r d \theta=\iint_{A} 2 \pi r \psi d \theta$. Therefore, the factor $2 \pi r$ appears in the definition of the discrete operators above.

Solving the governing system (Eqs. 8, 9) monolithically (i.e. for velocity and pressure simultaneously) is a computationally tedious procedure. On the other hand popular pressure segregation or fractional step method (proposed independently by [24] and [25]) may lead to mass conservation problems [26], [27]. Thus, an efficient yet accurate modified fractional step strategy with improved mass conservation characteristics is implemented. The rationale of this strategy and the implementation details can be found in [26], [28]. Alternative strategy suitable for the problem of interest and based upon the accelerated monolithic approach can be consulted in [29].

\subsection{Contact and boundary mesh}

Contact modelling We implement here a simple algorithm that allows preserving the overall strategy of the PFEM, but considerably improves the accuracy in the prediction of the wall thickness. The algorithm introduces boundary markers and permits maintaining the unconstrained version of Delaunay triangulation. Unconstrained triangulation is computationally "cheaper" than the constrained one. Moreover, it facilitates contact detection.

Modified PFEM contact algorithm Let us consider that the nodes follow a variable $h(x)$ distribution, where $h(x)$ is the minimum distance between two nodes. Alphashape technique (see [30], [10]) applied to the unconstrained Delaunay mesh allows to distinguish whether the element consisting of the given three nodes must be created or not. The radius $r$ of a circle defined by the examined nodes is compared against the corresponding size $h$ (average size of the inter-nodal distances). If the ratio $\frac{r}{h}>\alpha$, where $\alpha$ is the alpha-shape parameter typically taken as 1.5 in $2 \mathrm{D}$, the element is not created and the nodes are marked as boundary (For further details on the application of alpha-shape technique in PFEM one can refer to [10]. A study of different alpha-shape values upon the accuracy of the method can be found in [19]). In order to accurately model the contact between the evolving glass domain and the fixed mould walls we propose to combine the alpha-shape technique with the boundary markers.

Let us mark the outer boundary of the glass domain and the mould wall with interface and wall flags, respectively. As the interface nodes approach the wall nodes, the distance between them diminishes and an element is created. Clearly, such element is non-physical in the sense that it creates a "premature" contact between the glass and the wall. This implies introducing an error of the order $h$ in the determination of the thickness.

Thus, we propose to treat such an element as a purely geometrical entity, that does not contribute to the governing equations. This can be formally described 


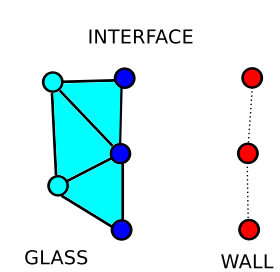

(a) Glass and wall are separated

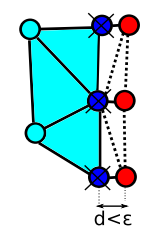

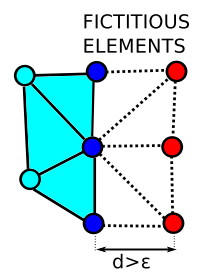

(b) Fictitious contact elements are created

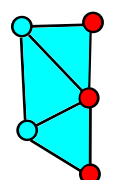

(c) Removal of interface nodes close to the wall (d) Stick contact between the glass and the wall

Fig. 3 Schematic representation of contact algorithm

as: if an element contains both interface and wall nodes, mark it as a fictitious element and multiply its contribution (residual and tangent matrix) by zero during Finite Element assembly process. Nevertheless, fictitious elements can be used for tracking the distance between the interface nodes and the wall. Moreover, creating fictitious elements as geometric entities enables maintaining the original PFEM unconstrained meshing strategy.

Next, the distance $d$ between the interface node and the wall is computed within each fictitious element. As soon as $d$ obtains a value lower than a given tolerance $\epsilon$, the interface node becomes erased, thus the glass domain gets connected to the wall via a real element in a fully sticking contact. This way, the error in the determination of the thickness of the bottle becomes controlled by the user-defined $\epsilon$. In the simulations used in the present work, $\epsilon$ was taken as $10 \%$ of the element size.

\subsection{Solution algorithm}

To this end all the "ingredients" of the model are defined. Table 1 presents the overall solution algorithm for the bottle forming process.

1. Discretize the glass domain with a finite element mesh.

2. Identify the external boundaries for the glass domain as well as the glass-mould wall contact elements (using the alpha-shape technique + boundary markers)

3. Solve the Lagrangian axisymmetric equations for the glass. Obtain nodal velocities, pressure and displacements.

4. Move the mesh nodes to a new position (according to the computed displacements).

5. Re-generate the mesh for the glass domain (nodes are generally maintained)

6. Go back to the next time step. Start the solution from Step 2

Table 1 Axisymmetric PFEM strategy for the bottle forming simulation 

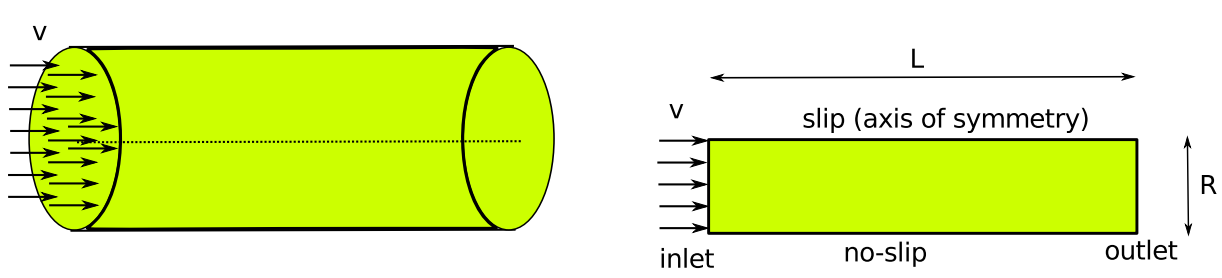

Fig. 4 Flow in a circular pipe. 3D and axisymmetric representations.

\section{Examples}

In this section numerical examples are solved. First example is used for the validation of the proposed axisymmetric PFEM formulation. Second one shows the functionality of the method in application to bottle forming simulation. Importance of using the proposed contact correction scheme for accurate mass conservation of the method is emphasized.

\subsection{Pipe flow}

This example models laminar flow in a circular pipe (see Fig. 4). This setting is perfectly axisymmetric thus serving as an excellent validation example for the proposed formulation. In the PFEM the flow inlet can be modelled by injecting the nodes. The nodes that move beyond the considered computational domain are deleted. The following geometry is considered: pipe length $\mathrm{L}=2 \mathrm{~m}$, pipe radius $\mathrm{R}=0.1 \mathrm{~m}$. Properties of the fluid are: density $\rho=1000 \mathrm{~kg} / \mathrm{m}^{3}$, dynamic viscosity $\mu=1000 \mathrm{~Pa} \cdot \mathrm{s}$. Gravity is neglected. Uniform inlet velocity is set to $v_{i}=0.1 \mathrm{~m} / \mathrm{s}$.

The analytic solution to the given problem is the parabolic velocity profile (paraboloid in 3D) that can be expressed as:

$$
v(r)=\frac{1}{4 \mu} \frac{\Delta P}{\Delta z}\left(R^{2}-r^{2}\right)
$$

where $\mathrm{r}$ is the distance from the symmetry axis.

Fig. 5 shows the velocity and the pressure profiles obtained in the numerical simulation using the proposed axisymmetric model. One can appreciate that the velocity profile is constant in axial direction (except for the regions in the proximity of the inlet and the outlet). Constant pressure gradient is $\frac{\Delta p}{\Delta z} \approx 75900$.

Fig. 6 shows the velocity along the "cut" made at $\mathrm{z}=1 \mathrm{~m}$. Result of the numerical simulation is compared with the analytic solution given by Eq. 16. One can see that the solutions are coincident ${ }^{2}$.

\footnotetext{
2 The maximum velocity can be also estimated by noticing that the volume of the paraboloid defined by the velocity profile must be equal to the area of the inlet multiplied by the inlet velocity. Taking into account the no-slip condition applied at the area where the constant non-zero flux is applied is $A_{\text {flux }}=\pi(R-h)^{2}$, where $h$ is the mesh size. Thus: $V_{\text {paraboloiod }}=$ $v_{\max } \frac{1}{2} \pi R^{2}=\pi(R-h)^{2} v_{i}$. Thus (taking $\mathrm{R}=0.1, \mathrm{~h}=0.0025$ ) we obtain: $v_{\max } \approx=0.19 \mathrm{~m} / \mathrm{s}$.
} 


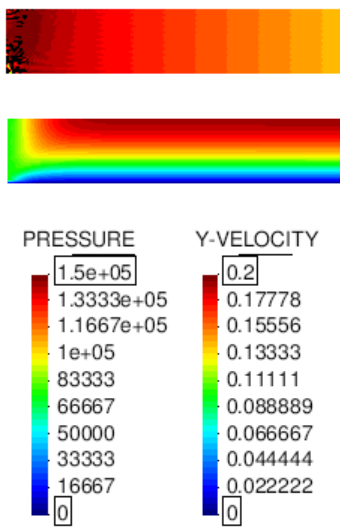

Fig. 5 Velocity and pressure fields at steady state in a circular pipe. Solution obtained using an axisymmetric PFEM model

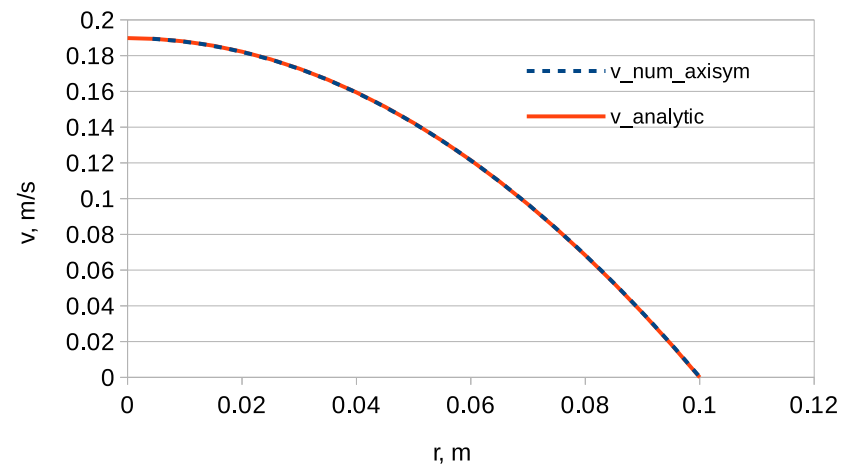

Fig. 6 Velocity profile across the pipe: comparison between the numerical results and the analytic solution.

\subsection{Bottle manufacturing: final blow process}

In this example the second stage of the bottle manufacturing process (involving the gravity stretching and the final blow) is modelled. The objective of this example is to test the capability of the presented approach to solving this challenging problem. In particular, proposed contact scheme and its impact upon the mass conservation is analyzed. For the sake of simplicity, only mechanical problem is solved and glass viscosity is considered constant. Clearly, this precludes the comparison of the obtained results with any experimental evidence. However, the results possess qualitative value. Moreover, the example and our results can serve as a benchmark for testing the mechanical modules of bottle forming models. It is worth mentioning that literature practically lacks reference data for the validation of bottle forming simulation. For non-axisymmetric problems the reader is referred to [7] for the benchmark examples.

The initial geometry consists of the mould wall and the parison. The geometry data is shown in Fig. 7. 


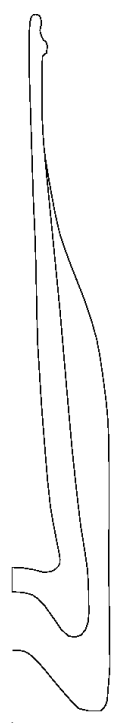

(a) Contour

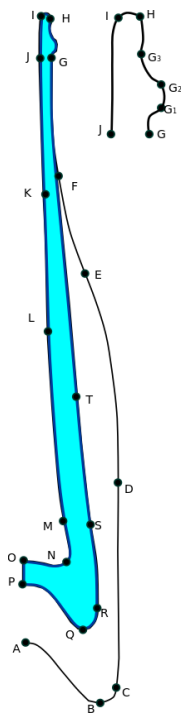

(b) Points

Fig. 7 Initial geometry of the the final blow set-up model

\begin{tabular}{|c||c|c|}
\hline Points & $\mathrm{r}$ & $\mathrm{z}$ \\
\hline $\mathrm{A}$ & 0 & -0.2815 \\
$\mathrm{~B}$ & 0.0346 & -0.30848 \\
$\mathrm{C}$ & 0.042 & -0.3020 \\
$\mathrm{D}$ & 0.0429 & -0.20632 \\
$\mathrm{E}$ & 0.0275 & -0.1127 \\
$\mathrm{~F}$ & 0.0163 & -0.0662 \\
$\mathrm{G}$ & 0.0144 & -0.0185 \\
$\mathrm{G} 1$ & 0.0163 & -0.0161 \\
$\mathrm{G} 2$ & 0.0153 & -0.01051 \\
$\mathrm{G} 3$ & 0.0136 & -0.006 \\
$\mathrm{H}$ & 0.0145 & -0.0014 \\
$\mathrm{I}$ & 0.0100 & 0 \\
$\mathrm{~J}$ & 0.0086 & -0.0185 \\
$\mathrm{~K}$ & 0.01004 & -0.0803 \\
$\mathrm{~L}$ & 0.01139 & -0.1420 \\
$\mathrm{M}$ & 0.01874 & -0.2241 \\
$\mathrm{~N}$ & 0.01963 & -0.2440 \\
$\mathrm{O}$ & 0 & -0.2447 \\
$\mathrm{P}$ & 0 & -0.2815 \\
$\mathrm{Q}$ & 0.028 & -0.2276 \\
$\mathrm{R}$ & 0.034 & -0.27 \\
$\mathrm{~S}$ & 0.029 & -0.22 \\
$\mathrm{~T}$ & 0.0246 & -0.169 \\
\hline
\end{tabular}

Table 2 Model for the final blow simulation 


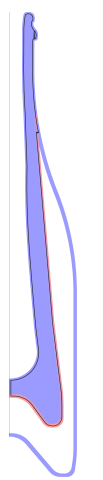

(a) 0 seconds

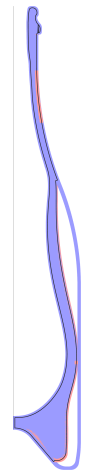

(e) 0.11 seconds

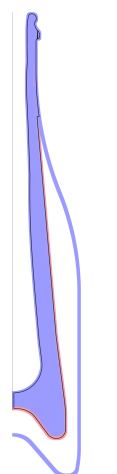

(b) 0.05 seconds

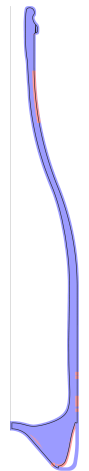

(f) 0.12 seconds

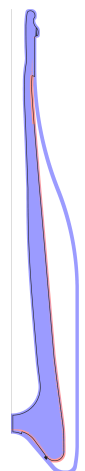

(c) 0.1 seconds

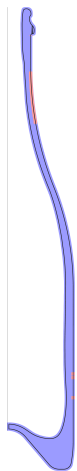

(g) 0.15 seconds

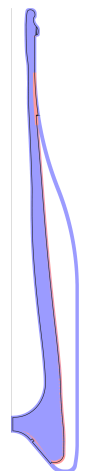

(d) 0.105 seconds

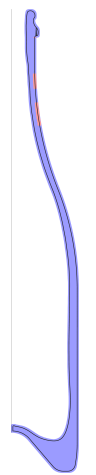

(h) 0.30 seconds

Fig. 8 Gravity stretching and final blow

The location of the points necessary for reproducing the geometry is shown in Fig. 7(b) and the corresponding coordinates are summarized in Table 2. The mould and the glass domain share the point $G$. The bottle neck and the mould are fixed. Domain boundaries are defined as follows: Dirichlet boundary $\Gamma_{d}$ comprises of the bottle neck and the mould wall.

During first 0.1 seconds the parison is exposed to gravity $\left(9.8 \mathrm{~m} / \mathrm{s}^{2}\right)$ exclusively. At $\mathrm{t}=0.1 \mathrm{~s}$ air pressure of $0.16 \mathrm{MPa}$ is applied at the inner cavity. An overall time $\mathrm{t}=0.3 \mathrm{~s}$ is simulated. Glass density is set to $\rho=2500 \mathrm{~kg} / \mathrm{m}^{3}$ and the kinematic viscosity is $0.65 \mathrm{~m}^{2} / \mathrm{s}$. The domain is meshed with nearly uniform unstructured mesh with a size of $0.0005 \mathrm{~m}$ leading to approximately 22000 linear triangular elements. This mesh size leads to a resolution of approximately 10 elements in the thickness direction of the glass domain.

Results Fig. 8 shows the evolution of the glass object. One can see that around 0.1 seconds glass reaches the mould bottom. The air pressure is applied then. It brings the glass in contact with the mould, first leaving a slight gap at the middle of the bottle height and the corner of the bottom $(t=0.11)$. At $t=0.12 \mathrm{~s}$ there remains only a small void at the bottom and afterwards the ultimate shape is obtained. It takes slightly more than $0.02 \mathrm{~s}$ to press the glass to the mould. 
The computational time necessary for performing the entire simulation was approximately 5 minutes (executed using 2 threads on a quad-core I7 PC).

Contact scheme and mass conservation Since the density of the glass is nearly constant, the mass conservation is directly proportional to the domain volume conservation. For an axisymmetric formulaton the domain volume is computed as $V=\sum 2 \pi A_{e l} \cdot r$, where $A_{e l}$ and $r$ are the area of an element and the radius of its centre, respectively.

Fig. 9 shows the evolution of the glass domain volume in time (The graphs correspond to a) actual domain volume obtained using the conventional PFEM contact algorithm b) actual volume corresponding to the modified contact algorithm presented here c) apparent volume of the present modified algorithm. Apparent volume is computed as a sum of all the elemental volumes (including "fictitious" elements). Actual volume accounts for the physical elements only. The exact value of the initial domain volume is $0.000347 \mathrm{~m}^{3}$. One can see that the standard PFEM contact algorithm leads to an increment in volume First, one can observe that standard PFEM contact algorithm leads to the volume increment of around $20 \%$ (area at $0.3 \mathrm{~s}$ is $0.00041 \mathrm{~m}^{3}$ ), while the proposed modified algorithm leads to an area change of less than $0.5 \%$ (the volume at the end of the simulation is $0.000348 \mathrm{~m}^{2}$ ). One can observe continuous increment in volume in case of standard PFEM contact algorithm when the new elements connecting the glass boundary and the mould wall are created. Maximum increment is observed after applying the air pressure as the entire outer surface of the glass is being brought in contact with the wall. A close-up of the contact region and the corresponding spurious elements are shown in Fig. 11.

Observing the apparent volume evolution of the presented contact algorithm one can see the increment in volume corresponding to the creation of the fictitious elements. These, however vanish as the actual glass boundary approaches the mould walls. Thus, at the end of the simulation all the fictitious elements become eliminated leading to coincident apparent and actual volumes.

This provides convincing evidence of the considerable improvement in the mass conservation provided by the very simple contact algorithm modification presented here.

Fig. 10 shows the wall thickness distribution along the vertical coordinate at $\mathrm{t}=0.3 \mathrm{~s}$. One can see a considerable difference in the thickness. One can see a considerable difference between the wall thickness distribution obtained with the standard PFEM and the modified contact algorithm. This result can be used as the main reference data for the future comparisons.

The spurious elements responsible for the inaccurate thickness are shown in Fig. 11.

Summary and conclusions In this paper we presented an axisymmetric version of PFEM developed specially for bottle forming simulation. No axisymmetric PFEM has been developed previously. A contact algorithm ensuring accurate mass conservation was presented and tested.

The model was validated using pipe-flow example. A numerical example dealing with the gravity stretch and final blow stage of the bottle manufacturing process was simulated. 


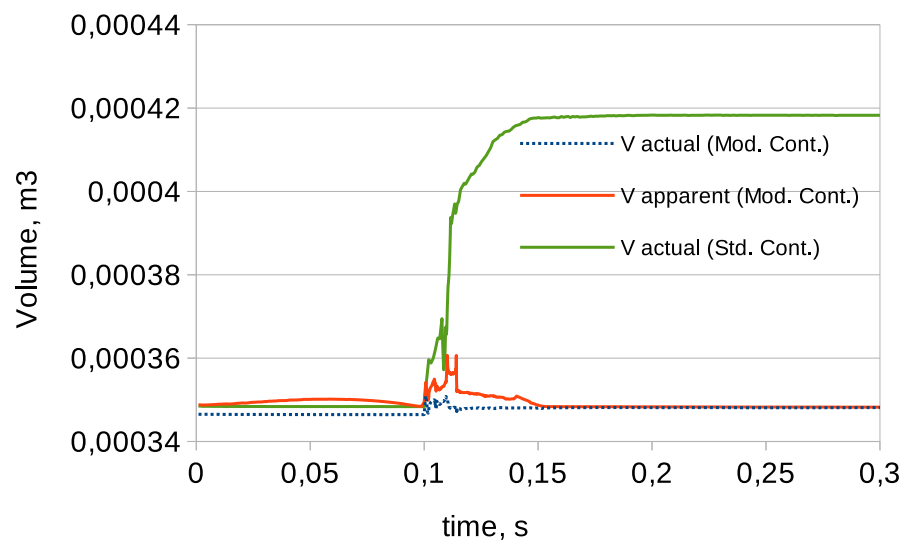

Fig. 9 Temporal evolution of glass volume

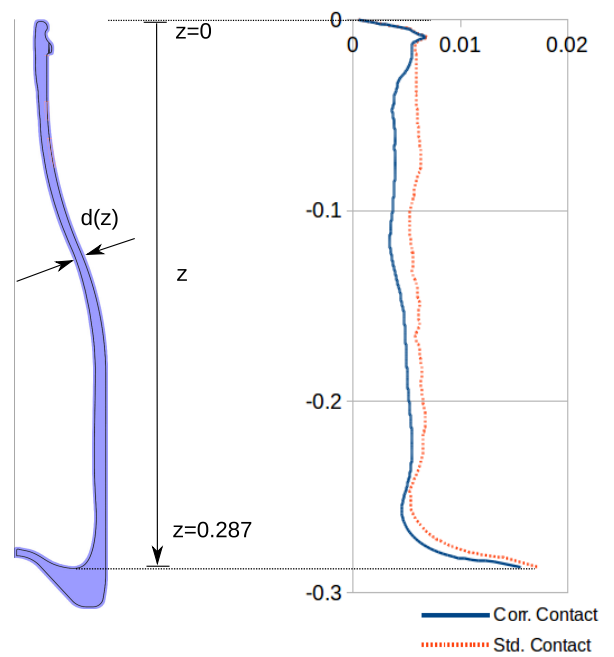

Fig. 10 Wall thickness distribution along the bottle height

The final blow process could be simulated in a very reasonable computational time (5 minutes using a quad-core I7 PC) on a computational mesh containing around 20000 triangular elements.

The facility of the boundary evolution tracking together with the relatively minor topology changes encountered in the problem of interest proves that Lagrangian approaches are more advantageous for the problem at hand than the Eulerian ones. The improved contact scheme presented here is extremely simple yet very promising, allowing the user to control the error in the thickness direction. In the present work the permissible error was set to $\approx 10 \%$ of the element size. The simulations carried out have proven that the method possesses excellent mass conservation features ( $\approx 0.5 \%$ of volume variation for the given contact tolerance) 


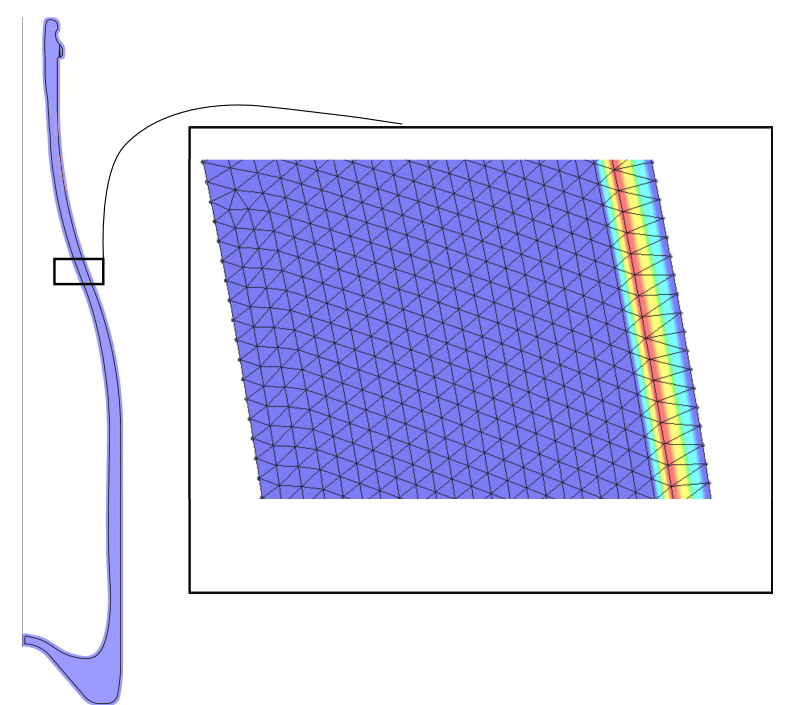

Fig. 11 Error in the thickness estimation when using standard PFEM contact scheme

in contrast with nearly $20 \%$ of mass variation in case of using the standard PFEM contact scheme (for the given mesh).

Two important steps must be carried out in the future. First is the implementation of the thermo-mechanical coupling. Once thermo-mechanical coupling is implemented, rigorous experimental validation of the method in application to bottle forming must be performed.

\section{Acknowledgements}

The author expresses his gratitude to the Spanish Ministerio de Economia y Competitividad for the FPDI-2013-18471 grant that allowed to perform this work.

\section{References}

1. H. G. Pfaender. Schott guide to glass. Springer Science \& Business Media, 2012.

2. G. L. Miller and C. Sullivan. Machine-made glass containers and the end of production for mouth-blown bottles. Historical Archaeology, pages 83-96, 1984.

3. R. Lerman. eBottles a history of glass bottle and glass jar manufacturing. https://www.ebottles.com/articles/GlassHistory.htm. Accessed: 2016-16-03.

4. J.M.A. Cesar de Sa. Numerical modelling of glass forming processes. Eng. Comput., 3:266-275, 1986.

5. H. Matthew. Numerical simulation of glass forming and conditioning. Journal of the American Ceramic Society, 85(5):1047-1056, 2002.

6. E. Feulvarch, N. Moulin, P. Saillard, T. Lornage, and J-M Bergheau. 3d simulation of glass forming process. Journal of materials processing technology, 164:1197-1203, 2005.

7. P. Ryzhakov, Garcia J., and Oñate E. Lagrangian finite element model for the $3 \mathrm{~d}$ simulation of glass forming processes. Computers and Structures, 2016. submitted.

8. NoGrid pointsBlow software. http://www.nogrid.com/product/nogrid-points-blow1. Accessed: 2016-16-03. 
9. S. Idelsohn, E. Oñate, and F. Del Pin. The Particle Finite Element Method: a powerful tool to solve incompressible flows with free-surfaces and breaking waves. International Journal of Numerical Methods in Engineering, 61:964-989, 2004.

10. E. Oñate, S. Idelsohn, F. Del Pin, and R. Aubry. The Particle Finite Element Method: an overview. International Journal of Computational Methods, 1:267-307, 2004.

11. P. Ryzhakov, E. Oñate, R. Rossi, and S. Idelsohn. Lagrangian FE methods for coupled problems in fluid mechanics. CIMNE edition, 2010.

12. P. Dadvand, R. Rossi, and E. Oñate. An object-oriented environment for developing finite element codes for multi-disciplinary applications. Archieves of Computational Methods in Engineering, 17/3:253-297, 2010.

13. Kratos multi-physcis. http://www.cimne.com/kratos/. Accessed: 2016-16-03.

14. T.P. Seward III and T. Vascott. High temperature glass melt property database for process modeling. Wiley-american ceramic society edition, 2005.

15. S.J. Osher and R. P. Fedkiw. Level Set Methods and Dynamic Implicit Surfaces. Springer edition, 2006.

16. C.W. Hirt and B.D. Nichols. Volume of fluid (VOF) method for the dynamics of free boundaries. Computational Physics, 39:201-225, 1981.

17. A.-K. Tornberg and B. Engquist. A finite element based level-set method for multiphase flow applications. Computing and Visualization in Science, 3(1-2):93-101, 2000.

18. B. Delaunay. Sur la sphere vide. Izvestia Akademii Nauk SSSR, Otdelenie Matematicheskikh $i$ Estestvennykh Nauk, 7:793-800, 1934.

19. A. Franci and M. Cremonesi. Critical investigation of the particle finite element method. part i: volume conservation with remeshing. Computational Particle Mechanics (CPM), 2016. submitted 19/02/2016.

20. J. Donea and A. Huerta. Finite element method for flow problems. J. Wiley edition, 2003.

21. O.S. Zienkiewicz, R.L. Taylor, and P. Nithiarasu. The finite element method for Fluid Dynamics. 6th Edition, 3 Volumes. Elsevier butterworthheinemann edition, 2009.

22. F. Brezzi and K.-J. Bathe. A discourse on the stability of the mixed finite element formulations. Journal of Computer Methods in Applied Mechanics, 22:27-57, 1990.

23. P. Ryzhakov, R. Rossi, S. Idelsohn, and E. Oñate. A monolithic Lagrangian approach for fluid-structure interaction problems. Journal of Computational Mechanics, 46/6:883-399, 2010.

24. N.N. Yanenko. The method of fractional steps. The solution of problems of mathematical physics in several variables. Springer edition, 1971. translated from Russian by T. Cheron.

25. A.J. Chorin. A numerical method for solving incompressible viscous problems. Journal of Computational Physics, 2:12-26, 1967.

26. P. Ryzhakov, E. Oñate, R. Rossi, and S. Idelsohn. Improving mass conservation in simulation of incompressible flows. Int. Jour. for Num.Methds. in Eng., 90/12:1435-1451, 2012.

27. S. Idelsohn and E. Oñate. The challenge of mass conservation in the solution of free-surface flows with the fractional-step method: problems and solutions. International Journal for Numerical Methods in Biomedical Engineering, 26:1313-1330, 2010.

28. P. Ryzhakov. A modified fractional step method for fluid-structure interaction problems. Revista Intern. Met. Num. Ing. (RIMNI), 2016. DOI 10.106/j.rimni.2015.09.002.

29. P. Ryzhakov, J. Cotela, R. Rossi, and E. Oñate. A two-step monolithic method for the efficient simulation of incompressible flows. International Journal for Numerical Methods in Fluids, 74(12):919-934, 2014.

30. N. Akkiraju, H. Edelsbrunner, M. Facello, Fu P., E. P. Mucke, and Varela C. Alpha shapes: definition and software. Proceedings of International Computational Geometry Software Workshop, 1995. 